\title{
Sensory Media: Multidisciplinary Approaches in Designing a Situated \& Mobile Learning Envi- ronment for Past Topics
}

\author{
http://dx.doi.org/10.3991/ijim.v6i3.2097 \\ G. Liestøl ${ }^{1}$, A. Doksrød ${ }^{2}, \check{S}$. Ledas ${ }^{3}$ and T. Rasmussen ${ }^{1}$ \\ ${ }^{1}$ University of Oslo, Oslo, Norway \\ ${ }^{2}$ Midgard Historical Centre, Vestfold, Norway \\ ${ }^{3}$ Vilnius University, Vilnius, Lithuania
}

\begin{abstract}
Handheld digital devices are rapidly increasing their sensory capabilities for registering multiple types of input, such as movement, orientation, position and touch, as well as light and sound. Mobile Augmented Rreality is one of the emerging forms of representation and expression that exploits these sensory media. In the following text we will present and discuss situated simulations as a type of indirect augmented reality. In a situated simulation there is approximate identity between the $3 \mathrm{D}$ environment as displayed on the screen and the user's real perspective on a given location. This makes it possible to create simulations of relevant objects and environments related to a specific place, for example, interpretations of its past. We present a situated simulation - Borrehallen - reconstructing a hall from the early Viking age according to recent archaeological evidence. The simulation shows the structures as they once might have been positioned in the old cultural landscape, including multimodal information about a series of aspects and objects. The simulation was tested and evaluated by Year Six school children, as well as a smaller group of senior citizens. In the article we approach the design and use of the application system from multiple angles based on its interdisciplinary conditions and constraints: humanities informed design; archaeology; 3D graphics and user studies. Finally, we describe and discuss the users' response to the various attributes of the application, and compare the main differences in experience, interpretation and attitude between the two user groups.
\end{abstract}

Index Terms-Mobile Augmented Reality, Situated Simulations, sitsim, Situated Learning, interdisciplinarity.

\section{INTRODUCTION}

Media literacy is constantly challenged and changed by its interactions with emerging technologies in the digital domains. Currently, mobile and location-based solutions are gaining new ground - or places, to be more precise in the media market. This is partly due to the fact that handheld digital devices, in the shape of smartphones and tablets, are rapidly increasing their sensory capabilities for registering multiple types of input such as: positioning where in the world the device is (by means of GPS, WiFi base stations and cell towers); orientation - in which direction it is pointed (applying a magnetometer/digital compass); movement - how it is moved and gestured (measured by the accellerometer and gyroscope), and of course haptic input - how the user touches and manipu- lates the phone's interface (via its surface, including screen and physical buttons). These come in addition to more traditional sensors for light (the phone's camera for recording still images and video), sound (using the microphone to register audio input) and radiowaves for wireless networking. The ongoing convergence of these hardware sensors (more will find their way into the handheld devices in the near future) give rise to the notion of sensory media.

In this article we will describe and discuss our experiences with development, testing and user evaluation of a particular application of sensory media, a publication and simulation platform we call situated simulations. A situated simulation (sitsim) is a type of mobile augmented reality, which has been under development and testing in educational settings over a period of several years. For a closer description of the system and its evolution, see: [1, $2,3,4]$. In a sitsim there is relative congruity between the user's perspective in the real world and the perspective into the virtual environment, which is displayed on the screen (see figure 1).

The emergence of sensory media and augmented reality presentation techniques creates opportunities for both museum mediation to the public at large as well as learning in more organized and formal settings. In cultural heritage and the museum sector there has always been a

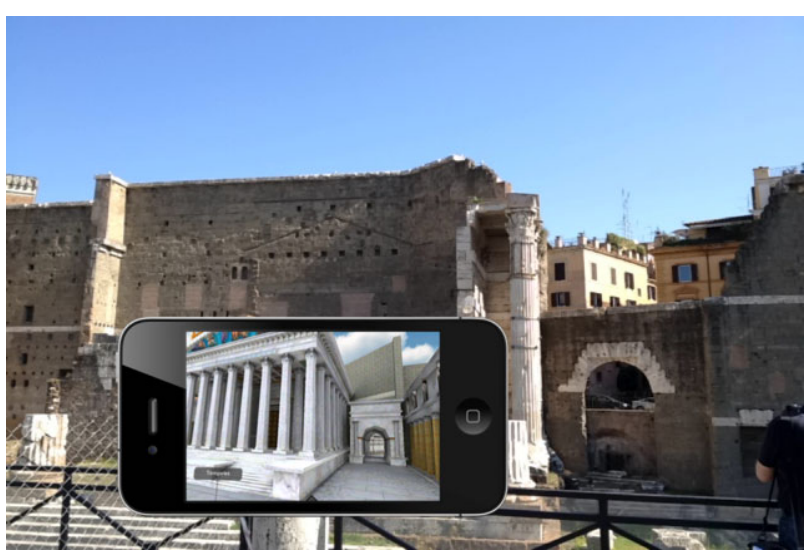

Figure 1. The congruity of real and virtual perspectives makes it possible to create alternative versions of a given environment, which can be viewed on location. Here, the reconstructed Forum of Augustus displayed on the phones screen and parts of the ancient remains in the background. The illustration is produced with the snapshot-feature of the application: using both the physical and the virtual camera. 
conflict between centre and periphery. Relics are removed from the original often peripheral site and collected and gathered in central locations. There, only to a limited degree, are they made available for access and public display. This creates both possibilities and problems. On the one hand, we see a concentration of competencies and improved preservation of artifacts as well as more effective mediations to larger audiences. On the other hand, local communities are drained of their historical traces when valuable objects are taken from their original context and relocated in a central institution. This situation is not only contradictory, but also paradoxical: museums secure the objects, but lack the context, while the local environment has the context, but the objects are absent. Mobile augmented reality and situated simulations may serve to remedy the conflict in this relationship. At the same time the on location virtual return of deported artefacts, knowledge and information creates new pedagogical opportunities for contextual and situated learning [5].

The research and development reported here is multidiscipinary in the sense that it combines a variety of knowledge fields, each possessing and practising different constellations of competencies and knowledge. They span from digital humanities and the use of rhetorical and narratological devices, via archaeological methods and museum mediation, to 3D graphic design and modelling, and last but not least user testing and evaluation in a media sociology perspective. These multiple perspectives have been necessary conditions and ingredients in the design and development process, and are thus reflected in the disposition and framework of this article. It is therefore important that the various perspectives are voiced in their own right and in a balanced manner.

In the following text we first present the archaeological subject matter and its background, the recently discovered Viking Age hall near the Borre grave field on the western shore of the Oslo fjord. Then we describe the pedagogical context of the local museum - Midgard historical centre and its involvement in the national programme for art and culture, a collaboration between professionals and Norwegian Schools (The Cutural Rucksäck/Den kulturelle skolesekken). Then the basic features of the situated simulation Borrehallen are portrayed with particular focus on how the virtual environment, including how its real time $3 \mathrm{D}$ objects and textures, are created and rendered. Then we go on to outline and explain the user testing, both with the school children and the senior citizens. Finally, we conclude with a general discussion and comparison of the user evaluations and relate these to further research considerations

\section{THE ARCHAEOLOGICAL TOPIC AND CONTEXT}

Within Scandinavia the Viking Period witnessed the transformation from tribal to state societies and a change of religions. Viking culture has contributed significantly to the creation of cultural coherence, symbolic values and cultural identity in the Nordic region, and it continues to hold immense public and popular appeal world-wide. This culture and its heritage evolved in close interaction within a unique natural environment.

Borre National Park is located in the Horten municipality in Vestfold, Norway. Here you can witness Northern Europe's most comprehensive assembly of monumental grave mounds from the Viking Age. There are seven monumental mounds, three stone mounds and about 40 smaller mounds in the park, all erected in the period between 600-900 AD. Until recently it was believed that this was the resting place of the Ynglings, one of the oldest known dynasties in Scandinavia.

To date, none of the remaining mounds in Borre National Park have been fully excavated. However, numerous archaeological surveys have been carried out in Borre, and interpretations of the material point towards Borre being a central place and a royal seat of power during the late Iron Age. If we consider the size of the monumental mounds it is quite possible that several of the deceased have been buried inside a ship, unfortunately there is little hope of retrieving any material that is not severely damaged.

Archaeological excavations in 1991 revealed traces of settlements alongside the Park's western stone fence; these findings were re-analyzed in 2007 using geophysical methods. Ground-penetrating radar (GPR) was used to send radar pulses to visualise the subsurface in the Borre Park. This non-intrusive method uses electromagnetic radiation, and detects the reflected signal from subsurface structures, and can detect objects, changes in material, voids and cracks. Using the GPR, archaeologists revealed several subsurface post holes, belonging to two big hall buildings. The images from the GPR showed that both halls had two rows of inner posts to support the structure, and solid wall constructions filled with vertical planks embedded in the ground. The walls of one of the halls were curved, making the building 10-12 metres wide in the mid-section and ca 8 metres towards the ends. The total length of the hall has been measured to be around 40 metres [6].

The two halls were both placed on the same contour line in the landscape, and pointing in the same direction. They could have existed during the same historical period, but it is also possible that they were used in two different periods. The GPR scan also indicated a possible building between the two halls, which indicates that there could have been several generations of houses built during a period that includes both Germanic Iron Age and Viking Age, i.e. the same period the grave field was in use.

In archaeology, hall buildings like the one in Borre can be identified among the house material from the 4th Century onwards. The building consists of one room, where the hearth was the source of heat and light. Artefacts found in these buildings indicate that the hall served an official purpose; common findings are elaborate glasses, weapons, gold and other objects clearly unrelated to the daily grind. The halls can be viewed as a ceremonial space, appropriate for expressing and maintaining the im-

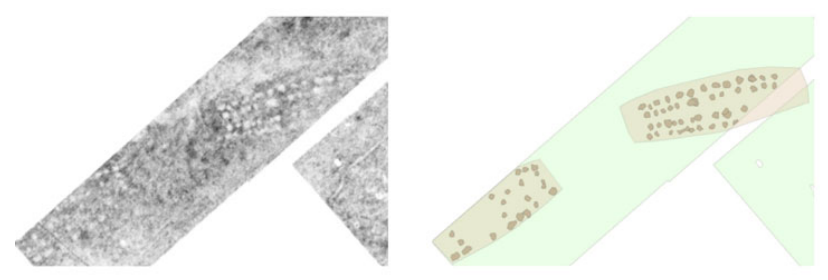

Figure 2. Image from the GPR, showing the subsurface anomalies of the northern hall building in Borre (left). To the right we see an image interpretation of the subsurface anomalies that constitutes the post holes of the two hall buildings (Photo, map and interpretation: UV Teknik, Swedish National Heritage Board). 
portance of family and lineage. The nature of findings from the halls is consistent with regard to this as they betray traces of seats of honour or thrones, and remnants of meals/feasts ands drinking rituals. It is very likely that weddings and funerals were held here, that this was where inheritance and property was handed down to the coming generations, and where alliances and networks were upheld [7].

In the Borrehallen sitsim we decided to reconstruct a large real/virtual environment: the whole landscape from the top of the great moraine down to the Viking Age sea level of the fjord, thus placing the halls and the grave mounds in their natural and strategic contexts. As a starting point we decided to target the sitsim towards public at large, but in particular the visiting school children.

\section{The CUltural Rucksack AT Midgard HistoricAl CENTRE}

The Cultural Rucksack (Den kulturelle skolesekken) is a national programme for art and culture provided by professionals in Norwegian schools. The programme helps school children to become acquainted with all kinds of professional art and cultural expressions. The objectives of the programme are to enable children and young people in primary and secondary school to enjoy artistic and cultural productions provided by professionals; to facilitate the pupils' access to a wide range of cultural expressions so that they can become acquainted with, and develop an understanding of, culture in all its forms; and to assist schools in integrating different forms of cultural expression with their own efforts to attain learning goals.

Midgard Historical Centre has been part of The Cultural Rucksack programme since 2001. Every year in May, Year Six students (12-year olds) from all the primary schools in Vestfold travel to Midgard to experience "a day in the Viking Age". The purpose of the day is to give the pupils the chance to use all their senses in learning about the Vikings and their life. The pupils are met by professional educators and the passing of knowledge is based upon the children's participation; they learn by doing, not just by listening.

The classes are divided into four different stations upon their arrival. During the day they rotate between the stations: Viking games, Role-play, Viking craft and trade, and archaeology. The pupils have 40 minutes in each station. All the activities take place outdoors, in Borre National Park and at the Viking playground outside the Midgard Historical Centre.

At the role-play station the pupils take part in a dramatization of daily life on a Viking Age farm. The dramatization is based on the storyline of a children's book, and the children are given the roles of the different characters in the book. The Viking craft and trade station gives the pupils the change to meet "real" Vikings and see old traditions come to life. At the Viking playground outside Midgard, the pupils gather to participate in various Viking Age games and activities. This includes learning how to shoot with a bow and arrow and throw axes at wooden targets/poles, trying to make them stand.

The final station is led by an archaeologist, and here the children get an introduction to archaeological fieldwork through participation in a simulated excavation. The children excavate the remnants of a "Viking house" from a large scale sandbox, and find artefacts that would nor-

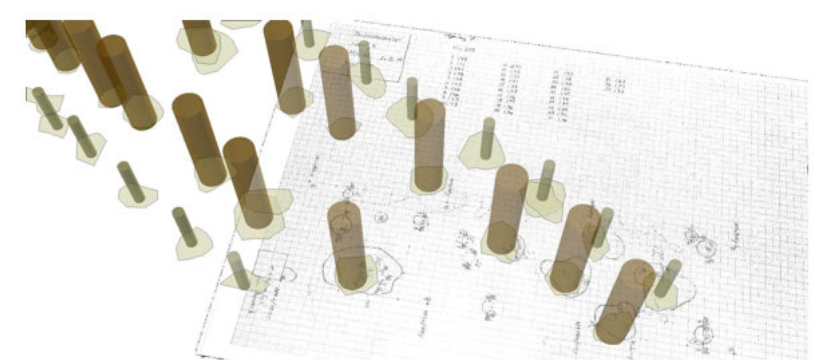

Figure 3. New interpretation of the findings from the archaeological excavation in 1991, then believed to be settlements, now interpreted as a feast hall (Image: Bjørn Myhre \& Terje Gansum)

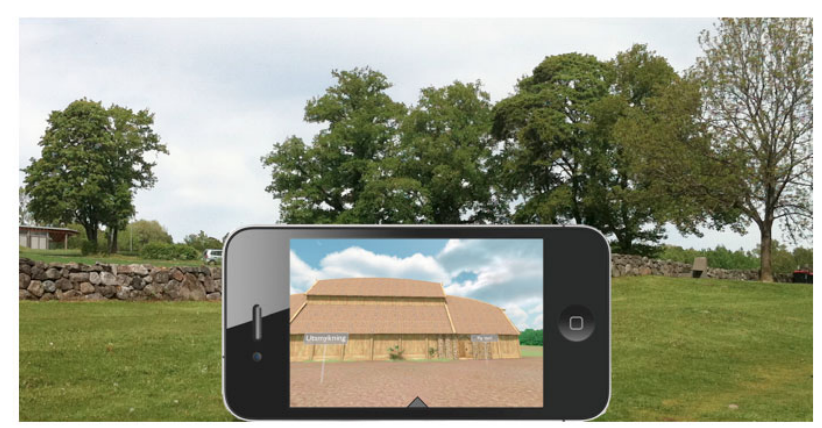

Figure 4. Illustration of Borrehallen in use point the device towards the North. The archaeological evidence was discovered immediately behind the stone fence in the background.

mally be found in a house from this period. From 2012 this station is called "Virtuell Vikingtid/Virtual Viking Age", and be replaced by the Borrehallen sitsim, focusing on the two hall buildings discovered in 2007.

\section{BORREHALLEN - THE BASIC FEATURES}

The Borrehallen sitsim builds on the several previous simulations of past topics, which we have designed and user tested over the last couple of years: in the Roman Forum, on the Acropolis in Athens, and at the Oseberg grave mound (see [3] and http://inventioproject.no/sitsim). The basic dynamics of Borrehallen is related to the three defining qualities of any mobile augmented reality application: (1) the combination of real and virtual objects in a real environment; (2) that the application runs interactively and in real time; and (3) that it registers (aligns) real and virtual objects with each other [8]. When using the Borrehallen app on location the virtual and the real perspectives are in parallel. This means that when the user changes position and moves the device in real space, the information on the display changes accordingly. In a way the screen then serves as a window where the user can look into another version of the local environments, in this case a reconstruction of the past (see Figure 4).

In addition to the basic dynamics of positioning, movement and orientation we included a series of functions available in the sitsim's feature set: links to additional information (audio narration, detail view of objects), transposition of a virtual camera relative to the user's real position, both vertically and horizontally (fly in, bird's view), and user generated links (pupils were given elementary assignments that required the creation, naming and positioning of a link including the assignment itself, the written answers to the questions (see figure 5). 

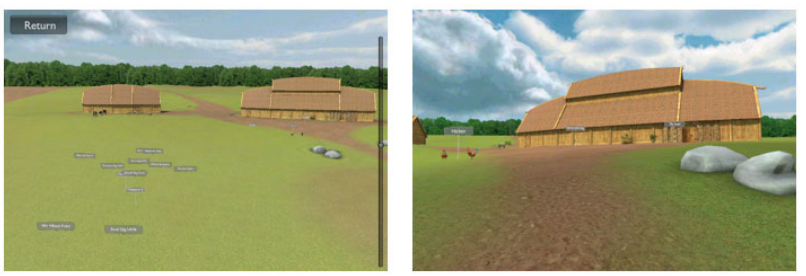

Figure 5. Screenshots from the iPad version of Borrehallen. The group of links (green border) positioned in front to the left are the assignments posted by one group of pupils. The links (grey border) in front of the larger hall building are system links. The perspective on the left is using the 'Bird's view' feature. The virtual camera is then positioned $12 \mathrm{~m}$ above the ground. Further adjustments of the altitude can be done using the slider to the right.

\section{CREATING THE ViRTUAL ENVIRONMENT}

Building the virtual environment is a crucial part of the simulation creation, since it directly affects the performance, usability and even the beauty of the application. Furthermore, this stage presents various challenges, some of which are related to the other parts of the process and some of which are quite separate. In this section we discuss the most important and sometimes complicated challenges creating the Borrehallen environment.

As in all real-time simulations or visualizations, the 3D environment in Borrehallen had to be optimal and adapted for real-time rendering. It could not have large amounts of polygons $^{\mathrm{i}}$, materials ${ }^{\mathrm{ii}}$ or textures ${ }^{\mathrm{iii}}$, so this had to be taken into account while modeling the scene. Since the application was targeted at newer iOS devices (iPhone4 and iPad2), it was decided to have around 50000 polygons in the scene. Another important aspect was the number of draw-calls ${ }^{\text {iv }}$ in the real-time rendering process, which usually depend on the texture and material count. It was decided that around 30 draw-calls should work well with the mentioned amount of polygons. However, the scene did not have large amounts of different objects, so even though the number of textures had to be taken into account, it was not the main issue. The polygon count, on the other hand, appeared to be a greater challenge.

The main building of the simulation had been recreated previousy for still image rendering. The polygon count of the original model was rather high, so it had to be reduced to an amount that resulted in good performance, but little loss of detail. Cleaning up and removing the unnecessary (or invisible) polygons helped a lot. On the other hand, merging some parts that use the same textures reduced the material count, which means that there would be less draw-calls in the scene.

The terrain was also one of the most detailed (in the sense of polygon count) objects in the environment, mostly because it had to cover an area of about 700 meters in diameter. To achieve a good result, the terrain was optimized in such a way that flatter or further areas had fewer polygons, whereas the more detailed or centred areas had more. Another issue was that the terrain could not be limitless, but the limits had to be hidden and not obvious to the user. In our interpretation the main area consists mostly of hills and grass fields, surrounded by forests. The forests have a rather evident contour, which gave us the possibility to cover the terrain limits with the tree line. The tree line, on the other hand, could be easily represented with a textured rectangle strip. Such a strip looks natural from a greater distance (See figure 6).

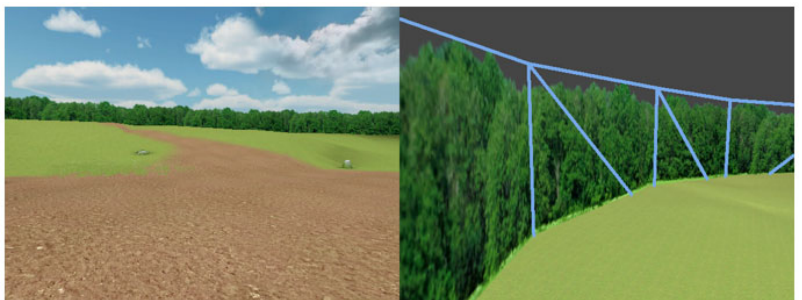

Figure 6. The view of the forest line from a distance (on the left), and from close up (to the right) with polygons selected and visualised.

However, the forest hid the terrain limits only on one side of the scene, not the other, which is the sea. Fortunately, this part was easy to make, because the water and its reflections cover the terrain. The forest almost reaches the water at both ends, so the whole environment becomes suitably bordered and hide its edges from the user. The water edge is obvious, but appears like a natural horizon. The empty space of the environment is then filled with a skybox, which blends well with the terrain and makes the scene more realistic.

The terrain creation introduced some other challenges as well. One of them was the modeling process itself and the effort to make it as accurate as possible. The landscape had to be built from a regular contour height map, which does not transfer to a 3D model. It had to be manually converted to a black and white height map, which could then be used as a displacement map. A panorama view, assembled from photographs of the area, was used to better understand the specifics of the terrain: the hills, slope and other details. The final displacement map was applied to a subdivided plane and the transformed model was saved as a new mesh. This way the displacement texture did its job, but was not directly needed anymore. The mesh was then automatically optimized by reducing the polygons as described before.

After making the terrain mesh, its material still had to be created and applied. Since the landscape is rather variable and needed to look natural, it was decided to use a custom multi-texture shader for the material. It enabled the usage of several overlapping textures that become more or less visible in some areas. There were two main textures and one additional. The two basic textures were grass and dirt and they added the colour and variety to the terrain. The images were tiling, which means that if you put copies of them next to each other, you cannot obviously see the edges of each tile. This way a large terrain model could be textured with quite small textures by simply repeating them. The colour of the two textures was made similar to the real ground colour in the photographs of the location. To make the two layers work together, the shader used the grass texture as a base and the dirt texture was used with its transparency information to distinguish the visible and less visible parts. The transparency, however, was not tiled, but scaled to the size of the whole terrain area. Since scaling the textures makes them blurry and the transition between grass and dirt did not need to be sharp, the low resolution of the transparency layer was adequate. The third layer of the terrain was used to make the whole scene livelier. In particular, it simulated shadows of moving clouds. This texture was mostly transparent and only some parts of it covered the terrain with semi-transparent black spots. The texture coordinates were animated (i.e. gradually changing over time), which made these pseudo-shadows move through the landscape. For 
this reason, the texture was also tiling, so it just repeated over time. With the described three layers it was possible to have a natural and lively landscape, which accompanied the main objects quite well. The result can be seen in figure 7.

So far we have described how the basic scene was built. But there was also a need to create some smaller details that would represent the specifics of the site. In particular, the wooden ornaments outside and inside the main building, some animals outdoors and several other objects inside. The most challenging were the ornaments: they could not be modeled because they would require too many polygons. Another issue was the need to recreate them from simple contours. The first problem was solved by using a texture that had depth and lighting information written into it. This was achieved by making a depth texture and then baking the lighting into a wood texture. (Figure 8 displays the process).

Going back to the essential decisions, it is worth mentioning that the scene did not feature characters or moving objects (except the animals that were animated, but not changing their position). Hence, there was no need to use real-time lighting, which would be expensive in the context of performance. It was decided to write the lighting and shadow information to the textures by using the lightmap baking functionality that the Unity engine provides. This made it possible to have realistic shadows and lighting, which even includes indirect lighting.

\section{USER TESTING AND EVALUATION WITH YEAR SIX STUDENTS}

In late May 2011, we tested the system at the Borre site, with the help of about 200 pupils from elementary schools in the region using iPad2. On the second day 88 of these were also asked to answer questionnaires, 43 boys and 45 girls, all 11-12 years old. The majority had mobile phones, but none of them smartphones. They all had experience with some sort of game console, such as PS or Nintendo, or they had either their own laptop or access to one. The laptops were used for computer games, homework or Facebook interaction. The most popular computer games were Sims 2 and 3, and game sites like Habbo and 123 spill. There were no differences between boys and girls regarding access to mobiles and ICTs. According to their own estimate, they spent between five and ten hours per week on the computer. The boys spent more time on their computer than the girls, particularly with soccer games and first person shooters like Call of Duty.

Before the trial, they were given a brief introduction to the Borrehallen sitsim. They were also given some easy assignments which required them to input written information to the system. Immediately after the trial, which lasted for about 30-40 minutes, they were asked to fill out a brief questionnaire. The following is a summary of the comments from the pupils.

Almost all the children expressed that they enjoyed the application. They commented that it was "cool", "super", "very nice", "exciting", "funny", and "well constructed". All the comments were very positive. Some added that it was a bit confusing, and not easy to know what buttons/links to press. On the question concerning what they liked the best, they replied that it was "cool" to see what had been on the site before, that the effects were "cool", that "when one turns, the system follows", "nice" that one"

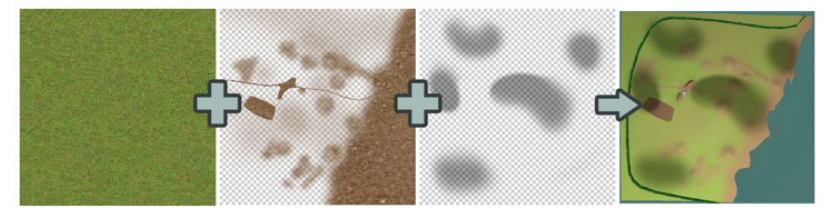

Figure 7. The first two images show the grass and dirt textures, the third one is the clouds and the fourth one is the result on an actual terrain (the checker pattern in the images displays their transparency information).

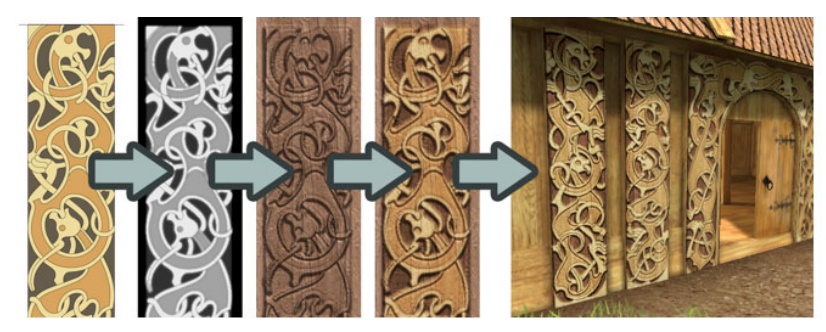

Figure 8. The first image shows one of the ornaments that the wood carver made. It only features lines for contours and some tones for basic depth information. The second image shows a depth map with edited edges to make them less sharp. The third picture displays the depth applied and the lighting baked into a wood texture (this was done in 3D modeling and rendering software). Finally, the fourth picture has some tones added to make it look livelier. The screenshot on the right side of the figure shows the finished texture applied to a $3 \mathrm{D}$ rectangle on the building wall.

could move around, "nice" that one could enter the building, "that one could enter the old days", "nice graphics", etc. They particularly enjoyed the 3D objects inside the hall that could be selected, turned, enlarged, etc. Most of the children would prefer more objects presented in this way. Also the "fly-in" function was "cool" and nice. They had no problem with looking around inside the building, even if they were dislocated from that position (due to the "fly-in" function).

What they liked the least, was that it was only one building, that it all was over rather quickly, and that the sound had uneven balance between the gain of the various audio types. The majority had however no problem with the sound level. Some "lost contact with the system" a few times, and needed help to continue. They all insisted that the system was either easy or fairly easy to use. Some commented that it was difficult to understand the system in the beginning.

The majority of the children said it was easy to grasp the function of the "links" or "signs" in the system, but some argued that they were difficult to understand, and that they would not have understood the purpose without an explanation. Also, the majority thought it was a suitable level of information about the topic in the system, although a substantial minority wanted more information on the Viking way of life. They enjoyed the simple assignment that they were given, and some would have liked more of that kind of activity.

The sound functioned well for the majority, but some argued that the level was too low and that it was difficult to understand what they were supposed to hear. On the question of what they heard, they confirmed the sounds embedded in the system: rain, thunder, hens, voices, birds. Almost all of the pupils thought the voice comments were easy to understand. Most of them did not miss more textual information in the programme. They would rather prefer more sound effects and audio comments. Some 
commented that it was difficult to see what happened on the screen because of the bright sunlight.

The pupils thought of the relationship between the screen reality and the physical reality as "cool" and "strange". Comments given were that it was "like steering a camera", "strange", "different", "exciting", and that it "looked real". On the question of whether they had learned anything from trying out the system, the majority replied that they knew more about what it looked like in the Viking Age, including how the trees, buildings and objects may have been. Even more of the pupils replied that they had learned to use an iPad, how to press buttons, and generally how to use a system like this. However, the majority also said they would have learned more from a film or an article on the subject. To describe the system, the children compared it with a computer game, Google street view, with Runescape, and Nintendo Wii. But the majority said that this was unique, they had never seen anything like it before.

\section{USER TESTING AND EVALUATION WITH SENIOR CITIZENS}

The Borrehallen sitsim was also tested with the assistance of eight senior members of the local community, from the hypothesis that elderly people may experience particular limitations (or particular advantages), due to their generational and sensory experiences. Their ages ranged from 65 to 85 years, with an average age of 75 . They were all interested in Viking history, but only moderately experienced in using smart-phones, tablets, apps and digital media in general. All had mobiles and PCs, but were inexperienced in using touch-phones and GPSmedia. The participants were given a brief introduction about the project and the technology with a focus on content and purpose: to experience the no longer existing Viking hall at the site where it was built more than thousand years ago. The information given about the equipment itself was fairly limited, since we wanted to test, among other things, the intuitive nature of the interface.

All participants were supplied with an iPad and earphones, and they were left to interact with the system individually. The textual information on the screen that introduces the application was considered informative and easy-to-read, if not of big interest due to some impatience to experience the simulation. When the graphical landscape appeared, the participants immediately understood the possibility to move around, approach the reconstructed hall and access information via the links (which they preferred to call road signs).

At this point, the main obstacle for the group appeared: The participants found it rather difficult to interact with the screen in order to receive information. Partly due to cold weather (and cold fingers), partly due to lack of experience in the use of touch-screens, several of the participants needed assistance with the touch-commands. Some insisted that using the thumb for touching worked better. Some of the participants found it awkward to hold the iPad in one hand and do the touch-commands with the other. Again, it was demonstrated that while the iPhone terminal is small, it is better suited for operating while walking [3].

In listening to the 'King' welcoming the participant into the hall, the participants suggested that more information could be received from the chief/king, possibly also from

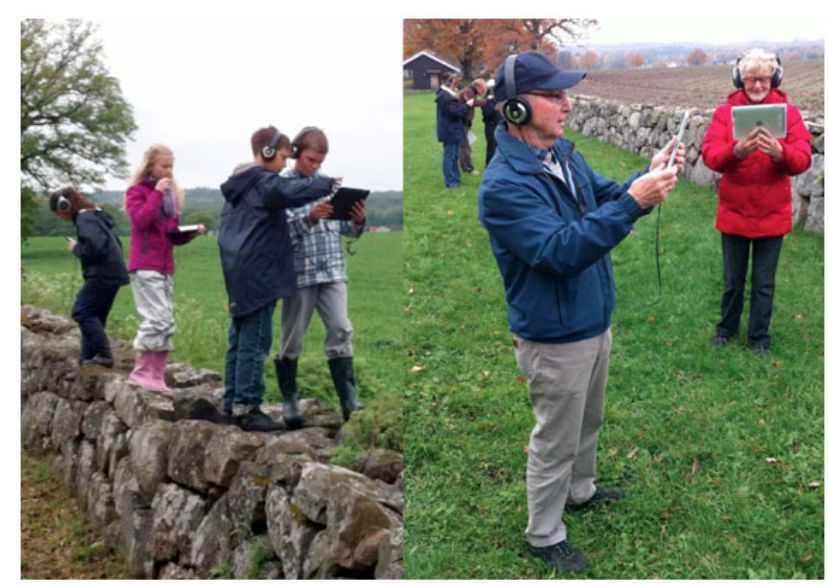

Figure 9. School children and senior citizens exploring the Borrehallen sitsim.

his wife. The possibility to focus on ornamental details was very welcomed, as they were very receptive to the beauty of Viking craft. The audio sound proved to be generally good, but the in-world sounds (rain, wind, hens....) were too low compared to the voice providing audio comments. The comments were considered informative if somewhat elementary for the group, more detailed information could have been provided.

It was a unanimous opinion among the group of elderly participants that the system was interesting to use and that it should have provided even more graphical information about the area. The group did not expect the system to replace human guiding, but considered that it could serve as a promising supplement to human guiding.

\section{CONCLUDING REMARKS AND FURTHER RESEARCH}

In comparing the experience of the two field trial groups there are several discrepancies, due to age differences and associated circumstances (knowledge, experience, interests, etc). While the group of senior citizens joined the trial partly because of their interest for Viking settlements and culture, the children did not share that motivation. They were more directed towards what was new, which was the technology itself. While the senior citizens would have liked more information in the system about the historical site, culture and daily life of the inhabitants 1200 years ago, the children were clearly more oriented towards the capacity of the system to simulate another reality. The two groups were exploring two different "realities". To use the vocabulary of Bolter and Grusin [8], they approached the remediation when using the simulation in a immediacy versus a hypermediacy way. The senior citizens, primarily interested in the subject matter, saw through the medium itslef, while the the school children were more focused on the mediation process itself, its new features and functions.

Despite this difference both the use and evaluation indicates that given further development mobile augmented reality in general and situated simulation in particular may serve as a new kind of learning tool employing new forms of multimodal competencies. The fact that it is virtually possible to relocate objects and environments, both in space and time, through this kind of simulation in situ, points to the relevance of situated learning [9] in this context. In further development of the system we will focus more directly at the learning potential, particularly 
for users to contribute with their own content, both in formal and informal settings.

\section{ACKNOWLEDGMENT}

We would like to thank the following for their valuable work making this project possible: Tomas Stenarson and CodeGrind AB, Matilde Liestøl, Lena Fahre, Bjarte Einar Aarseth, Magnus Liestøl and Richard Pilgrim.

\section{REFERENCES}

[1] Liestøl, G. (2009) 'Situated Simulations: A Prorotyped Augmented Reality Genre for Learning on the iPhone' in International Journal of Interactive Mobile Technologies (iJIM), Vol 3 (2009) - Open access available at $<$ http://online-journals.org/i-jim/article/ view/963 >

[2] Liestøl, G. \& Rasmussen, T. (2010) 'In the Presence of the Past. A field trial evaluation of a situated simulation design reconstructing a Viking burial scene' in Szücs, A. and Tait, A. W. (Eds.) Media Inspirations for Learning. Proceedings of EDEN 2010. Budapest: EDEN Secretariat, \% Budapest University of Technology an Economics 2010.

[3] Liestøl, G., Rasmussen, T. \& Stenarson, T. (2011) 'Mobile Innovation: Designing \& Evaluation Situated Simulations' in Digital Creativity 2011, Vol. 22, No. 3, pp. 172-184. Abingdon: Routledge, Taylor \& Francis Group (2011).

[4] Liestøl, G. (2011) 'Situated Simulations between Virtual Reality and Mobile Augmented Reality: Designing a Narrative Space' in B. Furth (ed.) Handbook of Augmented Reality. New York: Springer, pp 309-319. DOI 10.1007/978-1-4614-0064-6 14 $<$ http://www.springerlink.com/content/u457771n03602q1q/>

[5] Sharples, M., Taylor, J., Vavoula, G. (2005) 'Towards a Theory of Mobile Learning' in Proceedings of mLearn 2005. Available at $<$ http://www.mlearn.org.za/CD/papers/Sharples.pdf $>$
[6] Trinks, I. 2007. Borreparken. Archaeological prospection October 2007. UV Teknik. Stockholm: Swedish National Heritage Board, Archaeological Excavations Department.

[7] Gansum, T. 2009. 'Rurale strukturer, urbane funksjoner og definisjonsdiskurser' in Den urbane underskog. Oslo: Novus forlag.

[8] Azuma, R., Y. Baillot, R. Behringer, S. Feiner, S. Julier, B. MacIntryre, 'Recent Advances in Augmented Reality' in IEEE Computer Graphics and Applications, vol. 21, no. 6, Nov./Dec. 2001, pp. 34-47. http://dx.doi.org/10.1109/38.963459

[9] Bolter, J. and Gruisin (2000) Remediation. Understanding New Media. Cambridge: The MIT Press.

[10] Lave, J. and Wenger, E. (1991) Situated Learning: Legitimate Peripheral Participation. Cambridge: Cambridge University Press. http://dx.doi.org/10.1017/CBO9780511815355

\section{AUTHORS}

G. Liestøl is professor at the Department of Media \& Communication, University of Oslo, (e-mail: gunnar.liestol@media.uio.no).

A. Doksrød is archaeologist at Midgard Historical Centre, Vestfold, Norway (e-mail: anne.doksrod@vestfoldmuseene.no)

Š. Ledas is M.Sc. of Software Engineering at the Faculty of Mathematics \& Informatics, Vilnius University, Lithuania (e-mail: sarunas.ledas@gmail.com).

T. Rasmussen is professor at the Department of Media \& Communication, University of Oslo, (e-mail: terje.rasmussen@media.uio.no).

The research has been funded by Arts Council Norway and Department of Media \& Communication, University of Oslo. Manuscript received 23 April 2012. Published as resubmitted by the authors 24 June 2012.

\section{NOTES}

${ }^{\mathrm{i}} \mathrm{A} 3 \mathrm{D}$ polygon is a shape made of multiple vertices that are connected with edges, creating a closed chain (the inside of this chain is filled in and together with the vertices and edges makes the polygon). The $3 \mathrm{D}$ polygons are usually triangles.

${ }^{\text {ii }}$ A material is a set of information about the surface properties. It holds the textures, colours and also the information about how the surface reacts to light and other factors.

iii A texture is an image that is usually mapped on a 3D surface and is needed to add more colour, bumpiness or other similar details.

${ }^{\text {iv }}$ A draw-call is a single call to the graphics engine to perform the drawing of an object. Some objects might consist of multiple parts, which have to be drawn separately and therefore require multiple draw-calls (e.g. if those parts have a different texture). 